\title{
Towards a Fine-tunable Tibial Implant for the in-vivo Correction of Ligament Imbalance Resulting from Patient Morpho-functional Evolution
}

\author{
Shaban Almouahed ${ }^{1,2, *}$, Chafiaa Hamitouche ${ }^{1,2}$, Eric Stindel $^{2,3}$, Christian Roux $^{1,2}$ \\ ${ }^{1}$ Institut Mines - Télécom, Télécom Bretagne, 29238 Brest, France \\ ${ }^{2}$ Laboratoire de Traitement de l'Information Médicale, INSERM UMR 1101, 29609 Brest, France \\ ${ }^{3}$ Université de Bretagne Occidentale, 29200 Brest, France \\ *Corresponding Author: Shaban.Almouahed@telecom-bretagne.eu
}

Copyright (C)2013 Horizon Research Publishing All rights reserved.

\begin{abstract}
The main purpose of this paper is to summarize the results of our research to develop a new generation of instrumented knee implant. The proposed implant is supposed to provide the clinician with significant information on the tibiofemoral force distribution during normal walking in order to assess the ligament imbalance and correct it as needed. The electronics embedded into the implant to achieve this task should be miniaturized enough to fit the limited space available inside it. These electronics should also be self-powered to eliminate the need for periodic replacement of a rechargeable battery or the need for an external source of electric power. Therefore, the possibility to use the piezoceramics not only as imbalance sensors but also as energy harvesters within the tibial baseplate of knee implant has been investigated by means of theoretical modeling and experimental trials.
\end{abstract}

Keywords Instrumented Knee Implant, Tibial, Force Distribution

\section{Introduction}

Orthopedic surgery has significantly been evolved since the late $90 \mathrm{~s}$ with the advances in information and communication technologies. The computer-assisted surgical systems have recently been integrated in the operating room in order to aid the surgeon in planning and performing some surgical interventions. The integration of these systems has emphasized the need to develop patient-specific surgical procedures due to the fact that the conventional surgery does not take into account the patient-specific anatomy (repetitive gesture, identical for each patient). These technical advances in the knee and hip replacement surgeries have not been accompanied by equivalent advances in the im- plant design. The hip and knee prostheses implanted in the patients during sophisticated computer-navigated surgery, are identical to those implanted during the conventional surgery. The implant geometry is generic and does not take into account the anatomy of each patient. Approximately 50.000 Total Knee Arthroplasty (TKA) and 100.000 Total Hip Arthroplasty (THA) are annually performed in France [1] (In 2004, the most recent year with available estimates, there were approximately 454.000 TKA and 239.000 THA in the United States, up from 160.000 TKA and 117.000 THA in 1991 [2]). Approximately $71 \%$ of knee surgeries and $58 \%$ of hip surgeries are performed to patients aged 65 years old and over [2]. These proportions are related to the increasing number of gonarthrosis and coxarthrosis affecting persons over 70 years old. The population aging would be accompanied by a similar increase in orthopedic joint replacements. The aforementioned proportions demonstrate the socio-econo-medical importance of the addressed problem. Nowadays, the orthopedic prostheses are implanted while the patient is in supine position and under general anesthesia. This means that the surgery conditions are different from those encountered during daily life activities. In addition and after its implantation, the prosthesis will be subject to constraints evolving over time with many patient-specific factors such as age, weight and lifestyle. Accordingly, the intraoperative positioning and orientation of implant using multimodal information cannot be optimal in the absence of postoperative fine-tuning.

TKA represents a twofold surgical challenge consisting in obtaining the optimal compromise between the stability and mobility of prosthetic knee. To reach this compromise, the implant components should be precisely aligned with the mechanical axis of lower limb taking into consideration the stabilization functionality ensured by the knee collateral ligaments. The current designs of Total Knee Replacement (TKR) compensate for the absence of the anterior and posterior cruciate ligaments by 
the conformity of the prosthetic articular surfaces. On the other hand, the stabilization functionality is ensured by balancing the collateral ligaments during the surgical intervention. However, despite the fact that the cutting guides allow the surgeon to properly align the TKR components with the mechanical axis, there are no computerized systems to perfectly balance the collateral ligaments intraoperatively. Moreover, even if the collateral ligaments are perfectly balanced at the time of surgery, this balance will not necessarily remain perfect in the years following this surgery. This is due to the fact that the patient-specific morpho-functional factors will considerably change over time. Therefore, the functionality of TKR becomes suboptimal; which may sometimes lead to a revision surgery ( $9 \%$ at 10 years, $16 \%$ at 15 years, and $22 \%$ at 20 years) [3]. These proportions will result in a considerable number of revision surgeries taking into account the increase in life expectancy, on one hand, and the growing demand for this type of surgery by younger and active patients, on the other hand.

The purpose of our research is to develop a new generation of instrumented knee implant. Postoperatively, the tibial component of this implant should be able to compensate for the ligament imbalance by fine-tuning its adjustable shape. In order to achieve this goal, the tibial component must be instrumented with appropriate force sensors, efficient energy generator, low-power consumption telemetry system, and miniature actuation mechanism. The force sensors will be embedded into the tibial baseplate in order to measure the tibiofemoral force distribution needed to assess the ligament imbalance in the postoperative period. The telemetry system will be housed in the stem of the tibial component for data acquisition, processing, and wireless transmission. The actuation mechanism will be integrated between the tibial baseplate and the polyethylene insert for the postoperative fine-tuning of the implant's shape. The goal of the implant adjustment is to correct the ligament imbalance resulting from the morpho-functional evolution of the patient. The energy generator is indispensable for powering all the aforementioned electronics. The development of such an implant may eventually help in reducing the number of revision surgeries thanks to the implant's adjustability that enables the clinician to restore the perfect ligament balance when needed postoperatively.

\section{The obstacles that have been overcome}

During the postoperative follow-up visit, the proposed implant would allow the clinician to assess the ligament imbalance of the prosthetic knee and to correct it if needed. The most important challenge in developing such an implant is that of powering the electronics embedded inside it without the need for an external source of energy or even for an internal rechargeable battery. In other words, the instrumented implant should be selfpowered during its lifespan. Another challenge is to embed force sensors which are capable of measuring relatively high tibiofemoral forces acting on the tibial baseplate. These forces may attain a few times Body Weight
(BW) during most common activities of daily living. In addition, the sensors should be miniature enough in order to fit into relatively small space available within a custom-designed tibial baseplate without considerably increasing its thickness.

\subsection{The use of piezoceramics as forces sensors and energy harvesters}

In order to produce the electrical energy within the implant, the relatively high tibiofemoral forces produced inside the knee joint during daily life activities can be exploited. The most important activity of daily living is walking. Tibiofemoral compressive force acting on the tibial component during normal walking has been reported to vary between 1.7 [4] to $7.1 \mathrm{BW}$ [5]. This force can be a significant source of electrical energy when using the direct effect of piezoelectricity. In other words, the tibiofemoral forces can be converted into electric power by means of a few piezoelectric elements embedded into the tibial baseplate of the proposed implant. Furthermore, a judicious arrangement of these elements within the baseplate allows us to also use them as force sensors in order to assess the MedioLateral (ML) and AnteroPosterior (AP) imbalances of tibiofemoral forces. Using the same material to perform a twofold task enables us to better exploit the limited space available within the tibial baseplate.

\subsubsection{The first-version prototype and experi- mental trials}

An experimental prototype of the proposed implant has been developed for two purposes. The first purpose is to quantify the electrical energy that can be harvested by the embedded piezoelectric elements during simulated walking [6]. The second purpose is to validate the Center-of-Pressure (COP)-based approach as a means to assess the distribution of tibiofemoral forces among the four parts (AnteroMedial AM, PosteroMedial PM, AnteroLateral AL and PosteroLateral PL) of the tibial tray during the simulated walking and considering different levels of ligament imbalance [7]. The prototype was composed of femoral component and polyethylene insert provided by ADLER ORTHO Company ( $A D L E R$ ORTHO S. R. L., Milano, ITALY) in addition to the custom-designed tibial component developed in our laboratory (LaTIM, INSERM UMR 1101, Brest, France) (Fig. 1). The piezoelectric elements (SCMAP09 H4mm, Noliac, Inc.) were embedded within the tibial baseplate as shown in Fig. 2. The overall thickness of the tibial component (tibial baseplate, piezoelectric elements, and intermediate plate) was $6.65 \mathrm{~mm}$. This thickness is relatively small compared to that of the prototype developed by Platt et al. [8,9] (at least $22 \mathrm{~mm}$ ) knowing that a traditional tibial baseplate has a thickness of $4 \mathrm{~mm}$. The prototype was mounted and tested on a knee simulator (MTS 858 MINI BIONIX) operated according to the loading and displacement parameters recommended by the two International Standards (ISO 14243-1: 2002 and ISO 14243-3: 2004) [10,11]. Further details on the design of instrumented knee implant, used knee simulator, and measurement setup can be found in $[6,7]$. 


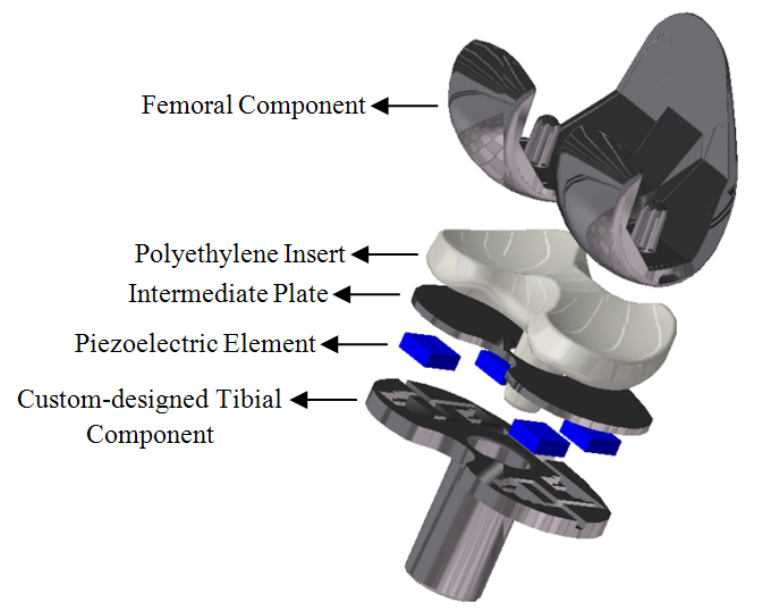

Figure 1: The experimental prototype of instrumented knee implant

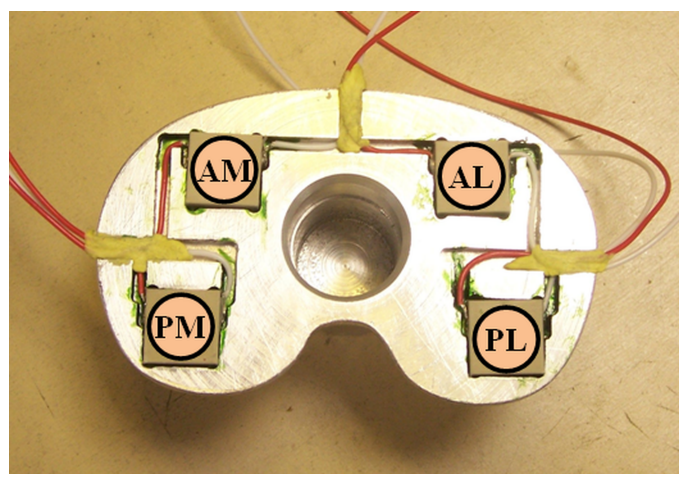

Figure 2: The piezoceramics disposed within the tibial baseplate

\subsubsection{The results of the theoretical and experi- mental studies}

Concerning the use of piezoceramics as imbalance sensors [7], the change in COP position with respect to a reference position can provide the clinician with significant information on the ML and AP distributions of tibiofemoral forces. Accordingly, the imbalance of collateral ligaments can be assessed, provided that the TKR components are perfectly aligned during TKA. The results obtained from the Finite Element (FE) modeling and experimental trials have shown clear relationship between the displacement of COP, either recorded by instrumented knee implant or computed by FE modeling throughout a simulated gait cycle, and the level of ligament imbalance represented by the medial offset of the axial force (Fig. 3). In conclusion, the results obtained from the experimental study confirm those obtained from the FE analysis study concerning the use of COP-based approach as an effective measure of ligament imbalance.

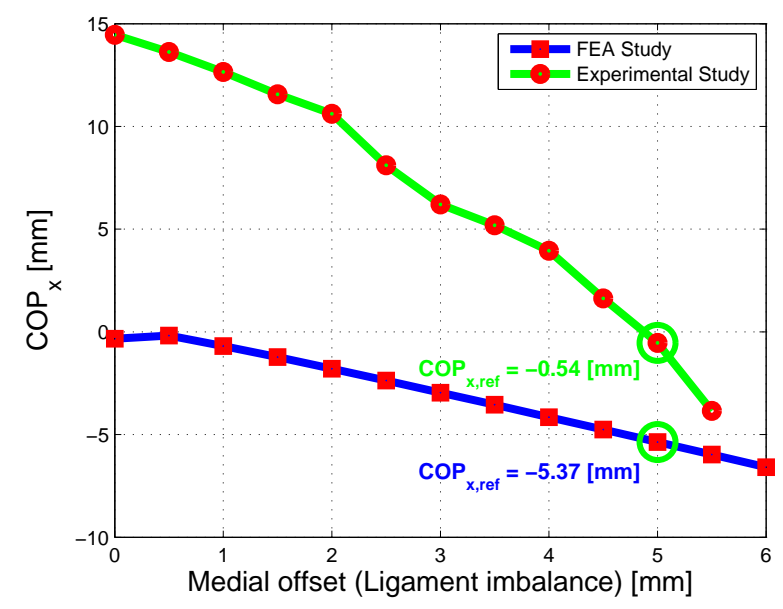

Figure 3: The average ML displacement of COP $\left(\overline{C O P_{x}}\right)$ as a function of ligament imbalance

Regarding the use of piezoceramics as energy generators [6], the amount of electrical energy that can be harvested by the piezoceramics embedded within the tibial baseplate has been quantified under operating conditions similar to those encountered in-vivo during normal walking. The energy quantification has been done using the developed electromechanical model and also using the experimental trials performed on the prototype during a gait cycle simulated by the MTS 858 MINI BIONIX knee testing machine. The electromechanical model has almost been validated by the experimental results which were consistent with the modeling results (Fig. 4).

\subsection{The development of low-power con- sumption telemetry system}

A low-power consumption system for data acquisition, processing, and wireless transmission is now being developed in collaboration with another laboratory ( $L a b$ STICC, CNRS UMR 3192, Brest, France) [12]. The telemetry system is supposed to be located in the stem 


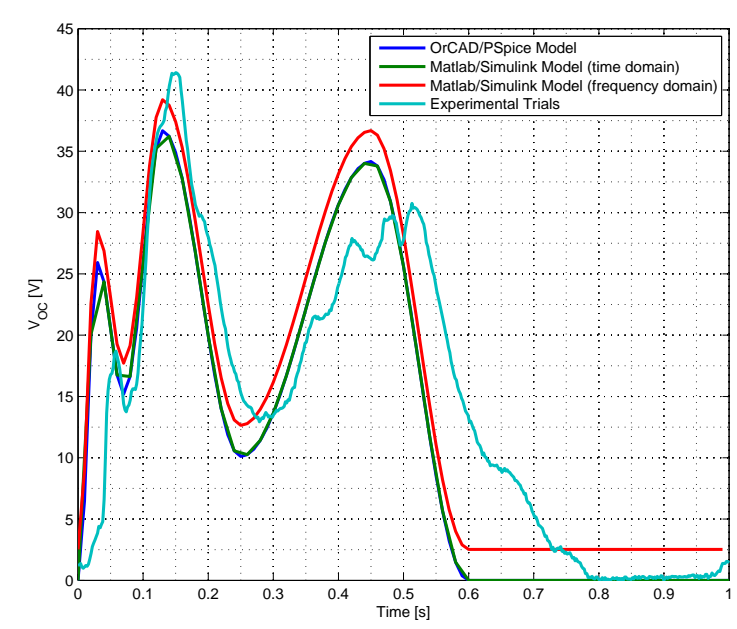

Figure 4: The overall open-circuit voltages obtained from the theoretical modeling and experimental trials

of the custom-designed tibial component in order to acquire and process tibiofemoral force data as well as to wirelessly transmit ligament imbalance information outside the knee implant when needed. This system will be powered by the electrical energy harvested using the piezoceramics embedded within the tibial baseplate.

\section{The obstacles that have to be overcome}

The main obstacles towards the development of an adaptive knee implant have deeply been discussed. The challenges concerning the energy generation, ligament imbalance assessment and wireless data transmission have been addressed and overcome. Theoretical modeling has been developed and experimental trials have been performed to validate a first-version prototype of the proposed implant. Nevertheless, the experimental prototype needs to be optimized in order to meet the in-vivo use requirements. An efficient power conditioning system should be considered to maximize the power transfer efficiency from the piezoceramic generator by matching and maintaining its output impedance. A miniature mechanism should also be designed, developed, and integrated into the custom-designed tibial component in order to correct the ligament imbalance as soon as it is assessed using the COP-based approach during the follow-up visit.

\subsection{The design optimization of the ex- perimental prototype}

The first-version prototype of the instrumented knee implant that we have proposed, developed and tested during the last three years was for experimental purposes. This prototype was used to investigate the possibility to generate electrical energy within TKR during walking and to quantify its amount. It was also used to explore how the different levels of ligament imbalance influences the COP position measured during a simulated gait cycle. In terms of calculations, the COP-based ap- proach used to assess the ligament imbalance was simple enough to meet the limited amount of energy harvested by the piezoceramics to power the entire electronic system including a microprocessor. Any increase in this amount would allow us to use more complicated, but more effective, approaches than that based on the COP. This complexity in the approach implies being energyhungry. Therefore, the design of the experimental prototype must be optimized in order to house piezoceramics having larger height and smaller cross-sectional area than those used in the first prototype without excessively increasing the thickness of the tibial baseplate where these piezoceramics are embedded. The purpose of using such piezoelectric elements is to harvest greater amount of electrical energy compared to that harvested by the elements used in the first prototype. The previously developed prototype cannot also be implanted into human body due to biocompatibility, sterilization, and lifespan issues. Hence, optimizing the prototype design in order to meet the in-vivo use requirements is an important challenge to overcome in the future research.

\subsubsection{Optimizing the design to increase the pro- duced energy}

The physical dimensions of a piezoceramic generator influence the amount of electrical energy that it can harvest. For a given input force, the mechanical stress (and consequently the mechanical strain) induced across the thickness of the piezoceramic generator depends on its cross-sectional area. In turn, the electric voltage that can be produced between the two electrodes of this generator depends on the mechanical stress. For a given resistive load, the harvested electric power is proportional to the square of the produced electric voltage. For an axial force such as that shown in Fig. 5 and a resistive load of $50 \mathrm{k} \Omega$, Fig. 6 shows how the average electric power changes with the cross-sectional area for four piezoceramic generators (SCMAP06, SCMAP0\%, SCMAP08, and SCMAP09, Noliac, Inc.) that have the same height $(4 \mathrm{~mm})$ but different cross-sectional areas $(3 \mathrm{~mm} \times 3 \mathrm{~mm}, 5 \mathrm{~mm} \times 5 \mathrm{~mm}, 7 \mathrm{~mm} \times 7 \mathrm{~mm}$, and $10 \mathrm{~mm} \times 10 \mathrm{~mm}$, respectively). In addition, the electric power produced by the piezoceramic generator depends on its height (Fig. 7).

For a piezoceramic, the smaller its cross-sectional area and the greater its height, the more the amount of electrical energy that it can generate considering the same load resistance and the same time-varying pressure. Nevertheless, any optimization of the prototype design in order to embed thicker and narrower piezoceramics should not involve an excessive increase in tibial baseplate thickness due to the fact that any increase will require the surgeon to perform excessive tibial bone removal in order to implant this baseplate. This, in turn, decreases the success rate of any possible revision of TKA. However, a 3D CAD model of an optimized prototype (acceptable by the orthopedic surgeon in terms of baseplate thickness) has been proposed and designed for further study in future work. As shown in Fig. 8.b, the piezoceramics (SCMAP08 H8mm, Noliac, Inc.) whose dimensions are $W \times L \times H=7 \mathrm{~mm} \times 7 \mathrm{~mm} \times 8 \mathrm{~mm}$ (Fig. 9) have been used instead of the piezoceramics 


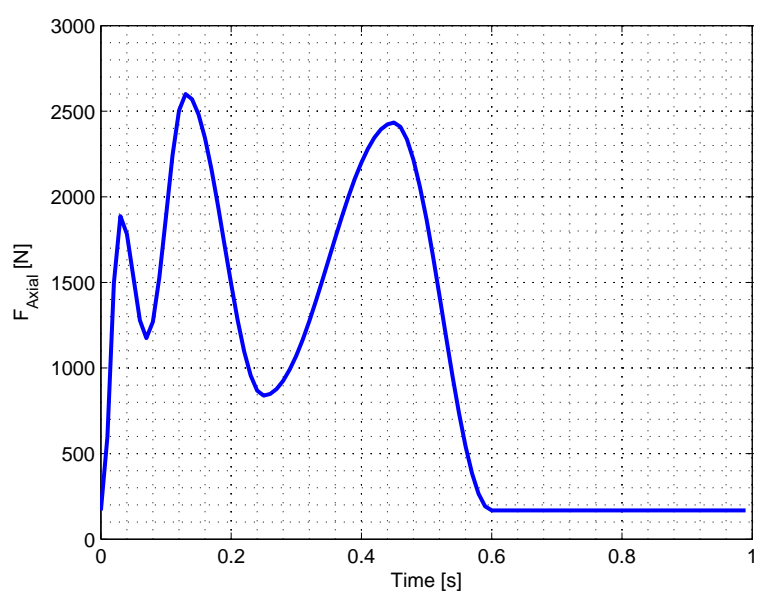

Figure 5: The axial tibiofemoral force experienced by the knee joint during walking [11]

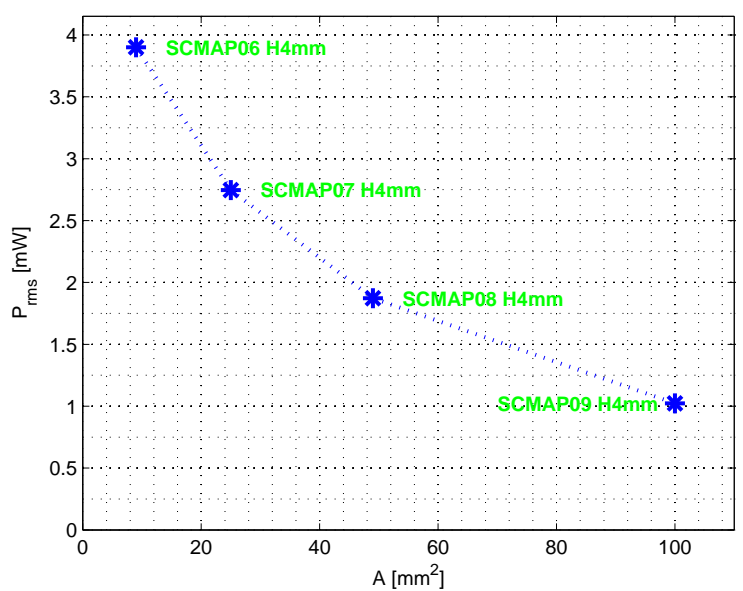

Figure 6: The change in the average electric power produced by the piezoceramic generator as a function of its cross-sectional area

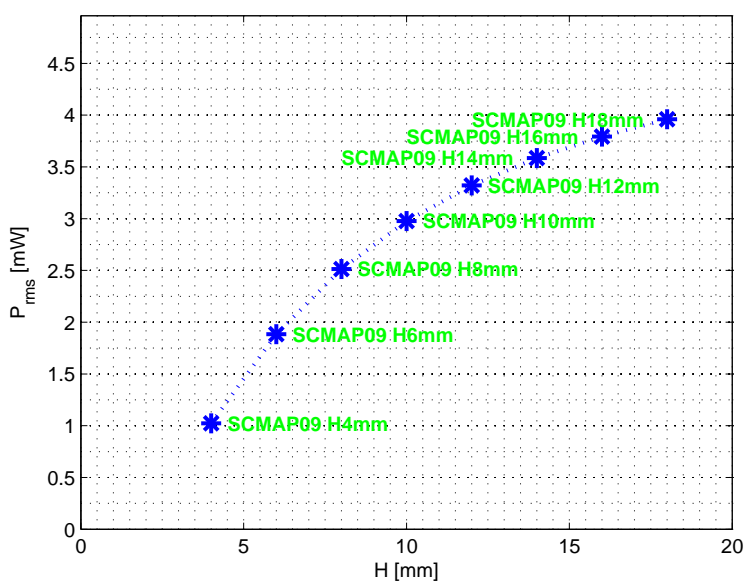

Figure 7: The change in the average electric power produced by the piezoceramic generator as a function of its height
(SCMAP09 H4mm, Noliac, Inc.) whose dimensions are $W \times L \times H=10 \mathrm{~mm} \times 10 \mathrm{~mm} \times 4 \mathrm{~mm}$. Therefore, four holes $(W \times L \times H=7 \mathrm{~mm} \times 7 \mathrm{~mm} \times 3 \mathrm{~mm})$ have been made across the thickness of the tibial baseplate and four corresponding cavities $(W \times L \times H=$ $7 \mathrm{~mm} \times 7 \mathrm{~mm} \times 2 \mathrm{~mm}$ ) have been added to the bottom of this baseplate in order to receive the lower ends of the SCMAP08 H8mm piezoceramics. Embedding such piezoceramics within the implant allows us to harvest a greater amount of electrical energy compared to that harvested by the piezoceramics used in the experimental prototype (1.023 $\mathrm{mW}$ across a resistance of $50 \mathrm{k} \Omega[6])$. According to the Matlab/Simulink model developed in [6], the average electric power $\left(P_{r m s}\right)$ harvested by a piezoceramic (SCMAP08 H8mm, Noliac, Inc.) and dissipated in a matched resistance of $34 k \Omega$ during a gait cycle is equal to $6.265 \mathrm{~mW}$.

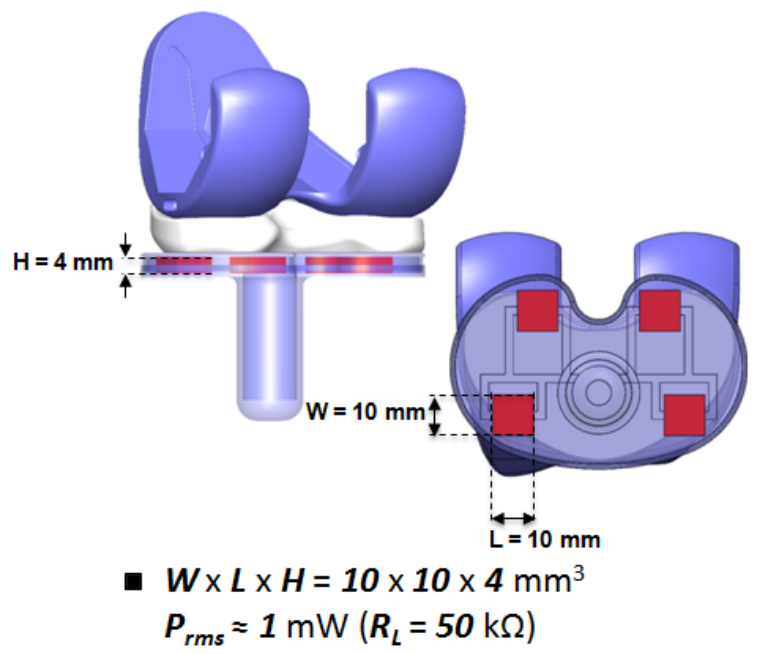

(a) Experimental prototype

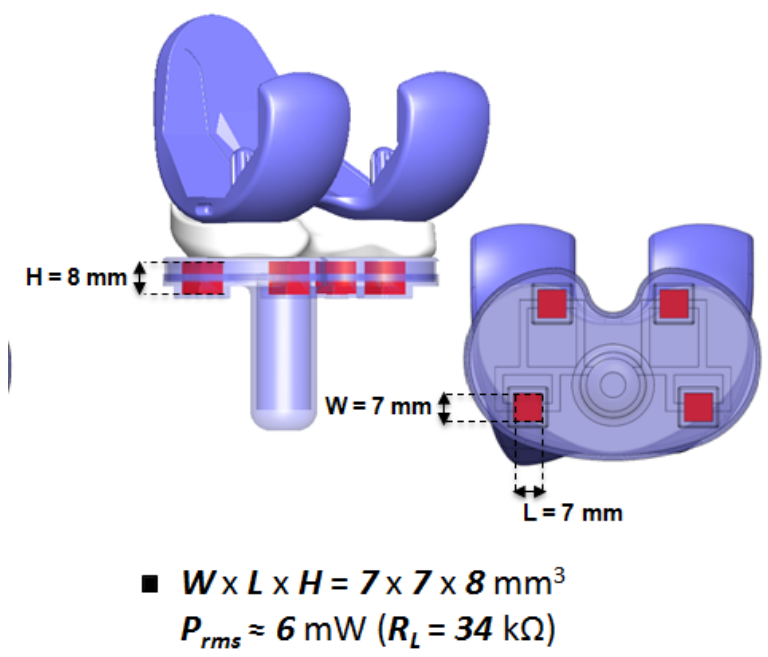

(b) Optimized prototype

Figure 8: 3D CAD model of the optimized prototype compared to the first prototype

\subsubsection{Optimizing the design to meet the in-vivo use requirements}

Another objective of the 3D CAD model proposed above is to demonstrate how it is possible to hermetically seal the electronics within the implant in order that 


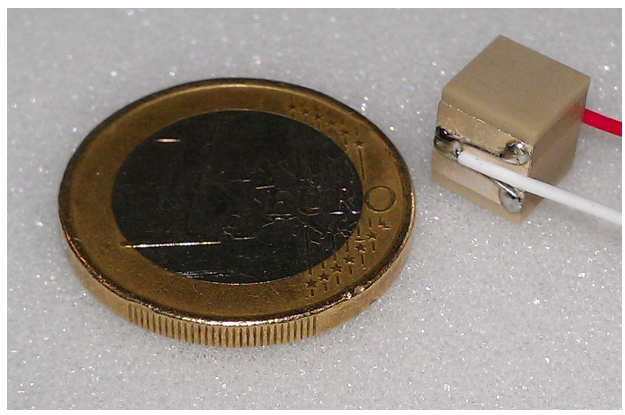

Figure 9: SCMAP08 H8mm, Noliac, Inc.

it fulfills the in-vivo use requirements. The customizeddesign of the tibial component has been modified so that the inside of the tibial baseplate where the electronics are embedded can be isolated from the outside by means of the intermediate plate which became like a top cover (Fig. 10). In this case, the non-biocompatible electronic components and circuitry can be packaged and hermetically sealed inside the implant in order to avoid any contact with the soft and bony tissues adjacent to the implant. Furthermore, the motion of the intermediate plate in the AP and ML directions is restricted while the inferior/superior motion is free within certain limits. The upwards and downwards motion allows the intermediate plate to vertically compress the piezoceramics without being in contact with the tibial baseplate. On the other hand, the motion restriction in the AP and ML directions reduces the shear forces and moments experienced by the piezoceramics during the 6-DOF knee motion and accordingly increases their structural lifetime. As a result, the longevity of the instrumented implant will be enhanced. For long-term implantable knee prosthesis, all the components that come into contact with the human body must be made of biocompatible materials having long-term bio-stability. Therefore, the femoral and custom-designed tibial components must be made of $(T i-6 A 1-4 V)$ titanium alloy. This alloy has very high stiffness compared with the tibial and femoral bones it replaces. Cortical bone has a stiffness of $15 \mathrm{GPa}$ and tensile strength of $90 \mathrm{MPa}$ [13]. Corresponding values for the titanium alloy are $110 \mathrm{GPa}$ and $860 \mathrm{MPa}$ [14], which are obviously very high. This provokes adverse bone remodeling and stress shielding, which over long-term leads to reduction in bone mass and implant loosening, particularly in the proximal bone-implant interface region.

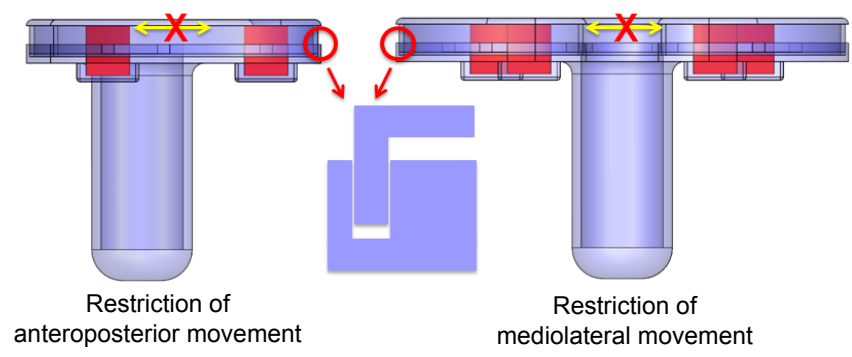

Figure 10: Restricting the AP and ML movements of the intermediate plate in the optimized prototype

\subsection{The development of an efficient power conditioning system}

Since the electronics integrated into the proposed implant needs a stabilized DC voltage to be powered and since the piezoceramic generator produces an $\mathrm{AC}$ voltage, the electrical output of this generator should be rectified, filtered, and regulated in order to ensure the electric compatibility. Therefore, one of the important obstacles to be overcome is the development of an efficient power conditioning system. This system is also being developed in collaboration with the aforementioned laboratory (Lab-STICC, CNRS UMR 3192, Brest, France).

\subsection{The development of a miniature ac- tuation mechanism}

An additional obstacle to be surmounted in the future research is the development of a miniature actuation mechanism. This mechanism integrated within the tibial baseplate may allow the clinician to correct any detected ligament imbalance by adjusting the height and inclination of the tibial tray (Fig. 11) according to the imbalance information provided by the piezoceramics. The fine-tuning of the tibial tray should be performed in the postoperative period in order to tension the lax ligament and consequently to restore the perfect ligament balance. This rebalancing should be performed without influencing the proper tibiofemoral alignment achieved during TKA. Different designs of a proposed mechanism have been studied using FE static structural analysis in order to predict the minimum lifetime under conditions close to those encountered in-vivo [15]. Tab. 1 summarizes the results for the design that fulfills the long lifetime expectations in case of in-vivo use. The actuator supposed to drive the fine-tunable mechanism is now under research and development through collab-oration between our laboratory and another laboratory (LIRMM, UMR 5506 CNRS UM2, Montpellier, France) [16].

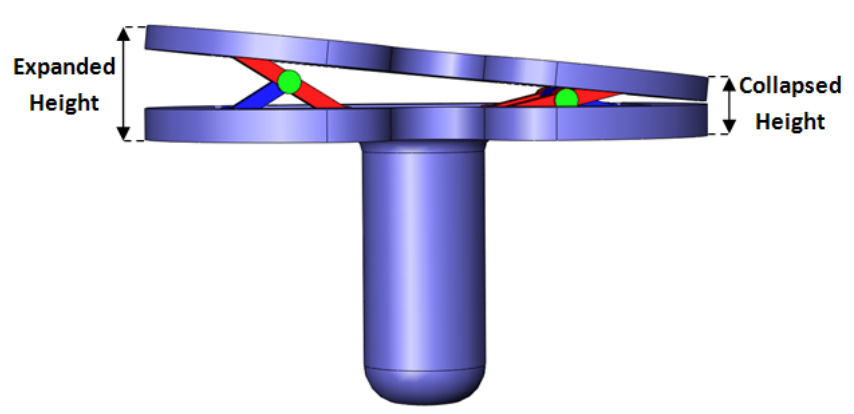

Figure 11: The adjustable tibial implant

\section{Conclusion}

In this paper, all the results of our research to develop an adaptive knee implant have been summarized. An experimental prototype of the proposed implant has been designed and tested to validate these results. Nevertheless, a final prototype must be considered after taking into account all the obstacles that need to be overcome. 


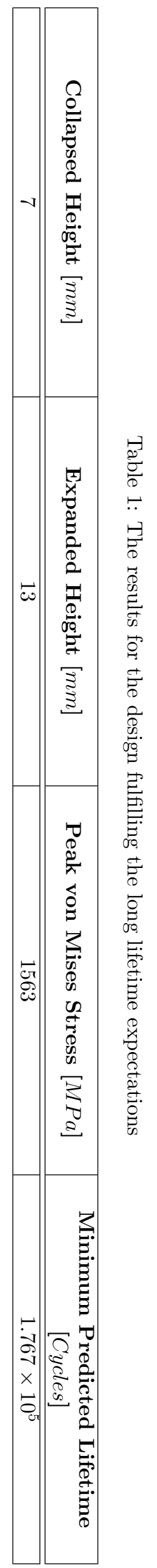


In the final prototype of the proposed implant (Fig. 12), the piezoceramic generator developed by our laboratory (LaTIM, INSERM UMR 1101, Brest, France) [6] will be used to produce an amount of electrical energy sufficient to power the low-power consumption system developed by the Lab-STICC ( $L a b-S T I C C, C N R S$ UMR 3192, Brest, France) for the acquisition, processing and wireless transmission of imbalance information to the outside of the prosthesis [12]. The postoperative ligament imbalance will be assessed via the original COP-based approach proposed by the LaTIM [7]. The assessed imbalance will be corrected by a miniature mechanical device being developed in collaboration between the LaTIM and the LIRMM (LIRMM, UMR 5506 CNRS UM2, Montpellier, France). The final prototype will be mounted and tested on a knee simulator that can realistically simulate the stabilization functionality of the collateral ligaments during various activities of daily life.

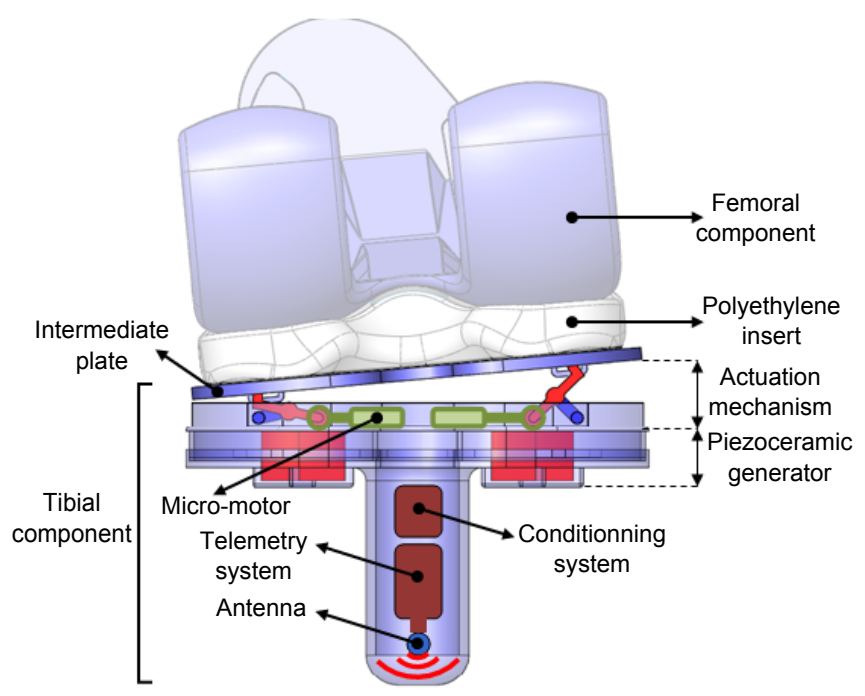

Figure 12: The fine-tunable knee implant with all the embedded systems

\section{REFERENCES}

[1] J.-M. Ziza, V. Zeller, N. Desplaces, and P. Mamoudy, "Infections sur prothèses articulaires : conditions du diagnostic et traitement," Revue du Rhumatisme, vol. 73, pp. 337-344, 2006.

[2] (2006, August) Orthopedics data compendium: Use, cost, and market structure for total joint replacement. IntegratedHealthcare Association. [Online]. Available: http://www.iha.org/

[3] J. A. Rand, R. T. Trousdale, D. M. Ilstrup, and W. S. Harmsen, "Factors affecting the durability of primary total knee prostheses," The Journal of Bone and Joint Surgery, vol. 85, pp. 259-265, 2003.

[4] R. D. Komistek, J. B. Stiehl, and D. A. Dennis, "Mathematical model of the lower extremity joint reaction forces using Kane's method of dynamics," Journal of Biomechanics, vol. 31, p. 185189, 1998.

[5] A. Seireg and R. J. Arvikar, "A mathematical model for evaluation of forces in lower extremeties of the musculoskeletal system," Journal of Biomechanics, vol. 6, pp. 313-326, 1973.

[6] S. Almouahed, M. Gouriou, C. Hamitouche, E. Stindel, and C. Roux, "The use of piezoceramics as electrical energy harvesters within instrumented knee implant during walking," IEEE/ASME Transactions on Mechatronics, Focused Section on Sensing Technologies for Biomechatronics, vol. 16, pp. 799-807, June 2011.

[7] S. Almouahed, M. Gouriou, C. Hamitouche, E. Stindel, and C. Roux, "Design and evaluation of instrumented smart knee implant," IEEE Transactions on Biomedical Engineering, vol. 58, pp. 971-982, June 2010.

[8] S. R. Platt, S. Farritor, and H. Haider, "On lowfrequency electric power generation with PZT ceramics," IEEE/ASME Transactions on Mechatronics, vol. 10, pp. 240-252, 2005.

[9] S. R. Platt, S. Farritor, K. Garvin, and H. Haider, "The use of piezoelectric ceramics for electric power generation within orthopedic implants," IEEE/ASME Transactions on Mechatronics, vol. 10, pp. 455-461, 2005.

[10] ISO 14243-1: Implants for Surgery - Wear of total kneejoint prostheses - Part 1: Loading and displacement parameters for wear-testing machines with load control and corresponding environmental conditions for test, International Organisation for Standardization Std. 14 243-1, 2002 .

[11] ISO 14243-3: Implants for Surgery - Wear of total kneejoint prostheses - Part 3: Loading and displacement parameters for wear-testing machines with load control and corresponding environmental conditions for test, International Organisation for Standardization Std. 14 243-3, 2004.

[12] C. Lahuec, S. Almouahed, M. Arzel, C. Hamitouche, M. Jézéquel, E. Stindel, and C. Roux, "A self-powered telemetry system to estimate the postoperative instability of a knee implant," IEEE Transactions on Biomedical Engineering, vol. 58, pp. 822-825, August 2010.

[13] J. Katz, "Orthopedic applications" in Biomaterials Science, B. D. Ratner, Ed. Academic Press, 1966.

[14] ASTM-F136: Standard specification for wrought Titanium-6 Aluminum-4 Vanadium ELI (Extra Low Interstitial) Alloy (UNS R56401) for surgical implant applications, American Society for Testing and Materials Std., 1998.

[15] S. Almouahed, C. Hamitouche, E. Stindel, and C. Roux, "Finite element lifetime prediction of a miniature adjustable orthopedic device," in $34^{\text {th }}$ Annual International Conference of the IEEE Engineering in Medicine and Biology Society - EMBC'12, 2012.

[16] A. Collo, S. Almouahed, C. Hamitouche, P. Poignet, and E. Stindel, "Towards a dynamic tibial component for postoperative fine-tuning adjustment of knee ligament imbalance," in $6^{\text {th }}$ International Conference on Biomedical Electronics and Devices - BIODEVICES 2013, Barcelona, SPAIN, 11-14 February 2013. 